\title{
Power and Spreading Factor Control in Low Power Wide Area Networks
}

\author{
Brecht Reynders*, Wannes Meert ${ }^{\dagger}$ and Sofie Pollin* \\ *Department of Electrical Engineering \\ ${ }^{\dagger}$ Department of Computer science \\ KU Leuven \\ Heverlee, B-3001 Belgium \\ Email: brecht.reynders@esat.kuleuven.be, wannes.meert@cs.kuleuven.be, sofie.pollin@esat.kuleuven.be
}

\begin{abstract}
Low power wide area networks are gaining interest to connect thousands of nodes to the internet of things. However, because the link budget in these networks is huge, nodes suffer from a near-far effect. Nodes far from the base station cannot send to the base station succesfully when closer nodes are transmitting, causing destructive collisions. LoRa, the considered technology in this paper, is a spread spectrum technology. It is known that spread spectrum is also sensitive to this effect. This paper presents a scheme to efficiently optimize the packet error rate fairness inside a LoRaWAN cell. This is achieved by optimizing the power and spreading factor for each node while avoiding near-far problems by allocating distant users to different channels. Simulations show that the packet error rate can be decreased up to $50 \%$ for edge nodes in a moderate contention scenario where 1 node per $1000 \mathrm{~m}^{2}$ transmits every 10 minutes.
\end{abstract}

\section{INTRODUCTION}

Low Power Wide Area Networking (LPWAN) is a promising technology to connect thousands of nodes to one single base station. More importantly, because of the high sensitivity of the base station, LPWAN enables connectivity to far locations where it used to be impossible. These features make LPWAN a key element for the Internet of Things. Examples of applications in need for long range technologies are asset tracking, smart grid and environmental sensing [1]. These applications consist of static or slowly moving nodes which typically send small payloads towards a server to process the data. LoRa with the LoRaWAN [2] MAC layer is an example of one of these emerging LPWANs.

\section{A. Motivation and related work}

The LoRaWAN network is a Chirp Spread Spectrum (CSS) based long range network. CSS is a spread spectrum technique where chirps are used to transmit the data. In this case, a number of bits is translated into a starting frequency, where the number of bits equals the spreading factor. This frequency is then linearly chirped to a maximal frequency, then continued from a minimal frequency until the initial frequency is reached again. Long range is enabled through the use of spreading factors which also enable simultaneous receptions of packets with different spreading factors. So, the higher the spreading factor, the longer it takes to transmit one symbol and the more robust, but also, the more bits in one symbol. The relation between the bit rate $R_{b}$, bandwidth $B$, spreading factor $S$ and symbol rate $R_{s}$ is summarized in Eq. 1 .

$$
R_{b}=\frac{B \times S}{2^{S}}=S R_{s}
$$

The nodes will adapt their spreading factor locally to the number of retransmissions or based on information embedded in the downlink messages from the base station. In [3], we showed already that letting the nodes choose the spreading factor and power control locally results in an unfair network with high packet error rates for nodes far from the base station. These nodes see significantly more collisions, increase their spreading factor to improve the link budget, but as a consequence only increase the number of collisions. In this paper, we propose a power and spreading factor control algorithm to mitigate this effect, that is to date still not solved.

Most papers show a spreading factor distribution scheme based on the distance to the base station ( [4], [5], [6]). In [6], they show that $28 \%$ of all nodes should use the highest spreading factor. This is necessary if the maximal range is needed, but, to the authors believe, cells will be much smaller to overhear packets on other base stations to enable location services. In these smaller cells, nodes use the lowest spreading factor if they base their spreading factor decision on link budget. However, we propose to change this distribution especially in smaller cells. Another reason to change this control is because these papers ignored the effect that different spreading factors have on each other, especially on longer ranges. A packet will drop if it collides with a much stronger packet with a different spreading factor without any control. In this paper, we show it is critical to combine power and spreading control to come to a meaningful link adaption strategy. To the best of our knowledge, power control has not been done in LoRa, but was rather used as a parameter to increase the potential range.

As described above, CSS enables decoding multiple messages with different spreading factors simultaneously. To decode simultaneous transmissions, power control is important because a threshold SNR needs to be guaranteed which is only possible when the received powers of all simultaneously transmitting nodes are of the same magnitude. Code Division Multiple Access (CDMA) is also a spread spectrum technique 
in which power control is a well investigated topic towards $3 \mathrm{G}$ cellular networks. Different algorithms exist: BER-based [7], SNR-based [8] and RSSI-based [9]. In our scenario however, we cannot use the SNR- or BER-based solutions, as they require fast feedback. In $3 \mathrm{G}$ networks, the update rate is $800 \mathrm{~Hz}$, while in LoRaWAN only one downlink message is available for each uplink message.

Finally, interesting research has been done concerning random access. [10] has shown the limits for random access networks with respect to retransmission probabilities and optimization of throughput given some failure constraints. This paper is different in the sense that the goal of our optimization is not throughput but packet error rate fairness.

\section{B. Contribution}

This paper has two main contributions. The first contribution is an analysis of a random access network with multiple spreading factors and rates. This paper calculates the optimal spreading factor distribution to use to minimize the collision probability. The second contribution is a scheme to distribute spreading factors and discrete power settings to nodes inside a LoRaWAN cell. The goal of this scheme is to improve the packet error rate for nodes far from the base station and hence, to create more packet error rate fairness inside a LoRaWAN cell.

The rest of this paper is structured as follows: in section II, the system model is described. This section also gives an introduction to the LoRaWAN MAC layer. Section III details the spreading factor distribution with and without limitations to the power control. Sec. IV proposes a scheme to optimize the settings for maximal packet error rate fairness and finally, some simulated results are given.

\section{SYSTEM MODEL}

This section describes the system model and assumptions used in the following sections. First, the lay-out of the cell is described and second, the necessary parts from the LoRaWAN MAC layer are explained.

\section{A. Cell lay-out}

In this work, a single LoRaWAN cell is considered. The cell contains one base station located in the cell center. Around this base station, $N$ nodes are placed with a uniform distribution over the area around the base station. The radius of the cell $R$ is considered to be small enough such that all nodes are able to reach the base station with every spreading factor and every power setting. The out-of-cell interference is ignored such that any frequency band can be used. The structure of this cell is identical to the one described in [10]. The nodes inside the cell generate small packets of data with size $L$. They generate one packet each $T_{i}$ seconds. The exact start of the packet is uniformly distributed over the entire slot of $T_{i}$ seconds.

Finally, the path loss model between the nodes and the base station is based on the log distance propagation model. This model is combined with a log-normal random variable $X$ to simulate shadowing gains. If a sensor in the networks sends with transmission power $P_{t}$, the base station received power $P_{r}$ is

$$
P_{r, d B}=P_{t, d B}-P_{0, d B}-10 n_{P L} \log _{10}(d)+X,
$$

where $P_{0, d B}$ is a constant power loss at 1 meter from the base node or base station in $\mathrm{dB}, d$ is the distance and $n_{P L}$ is the path loss exponent.

\section{B. LoRaWAN}

LoRaWAN [2] includes an open MAC layer built on top of CSS [11]. This MAC layer specifies 2 different traffic types of which only unacknowledged traffic is considered in the remainder of this work. The unacknowledged traffic consists of only uplink data packets with 1 downlink control packet within every 96 uplink messages to provide feedback about the uplink channel. These downlink messages will be piggybacked with the optimal power and spreading factor for the destination with negligible extra overhead.

The piggybacked data is the LinkADRReq MAC command from the LoRaWAN specifications [2]. This request is visualized in Tab. I. The overhead of this command is only 5 bytes. The information included is an ID field ( cid), the setting for the data rate (DataRate) and the setting for power (TXPower). Furthermore, it enables the control over all channels (Chmask and $C h M a s k C t l$ ) and it specifies the number of retransmissions for every packet (NbRep).

After each request, the receiving node answers with the 2byte long LinkADRAns MAC command, shown in Table II. The first byte is the command ID, while the second is a bit string where the last 3 bits are an acknowledgement for the data rate setting (DataRateAck), the power setting (PowerAck) and the channel mask (ChMaskAck).

Finally, it should be noted that LoRaWAN has limited choices available for power and spreading factor control. For power control, only 5 different values are available: $2,5,8$, 11 and $14 \mathrm{dBm}$. The spreading factor control, according to the LoRaWAN specifications, is limited to 6 unique spreading factors going from spreading factor 12 , or the lowest data rate, to spreading factor 7 , for the highest data rate. This means that up to 6 nodes can transmit simultaneously on the same channel.

\section{OPTIMAL SPREADING FACTOR DISTRIBUTION}

In this section, the optimal distribution of the spreading factor is derived to minimize collision probability in 2 different scenarios. First, the distribution is examined when power control is unconstrained, and then the practical case with 5 discrete power levels is considered.

\section{A. Distribution with unconstrained power control}

Given a network with unconstrained power control without limitations on transmit power, the transmit power counteracts the channel losses such that all nodes have the same received power. When considering multiple spreading factors, there are two types of collisions that can occur: (a) two packets with 


\begin{tabular}{c|c|c|c|c|c|c|c|} 
Bits & $0: 7$ & $8: 11$ & $12: 15$ & $16: 31$ & 32 & $33: 35$ & $36: 39$ \\
\hline Data & cid & DataRate & TXPower & ChMask & RFU & ChMaskCtl & NbRep \\
\cline { 2 - 7 } TABLE I \\
LINKADRREQ COMMAND AND THE LOCATION OF THE VALUES.
\end{tabular}

\begin{tabular}{c|c|c|c|c|c|} 
Bits & $0: 7$ & $8: 12$ & 13 & 14 & 15 \\
\hline Data & cid & RFU & DataRateAck & PowerAck & ChMaskAck \\
\cline { 2 - 5 } & \multicolumn{5}{c|}{ TABLE II }
\end{tabular}

LINKADRANS COMMAND AND THE LOCATION OF THE VALUES.

the same spreading factor collide, or (b) multiple packets with different spreading factors collide resulting in a bad signal-tointerference-noise ratio (SINR) for all packets involved. Note that the power control is assumed to ensure all packets have a good SINR at the base station in case there are no collisions.

a) Collisions with the same spreading factor: The probability of having at least one collision with the same spreading factor $S, p_{\text {coll }, S}$, can easily be written with the formulas of random access from [12], see Eq. 3 where $G_{S}$ is the amount of packets with spreading factor $S$ generated during the transmission of 1 packet with spreading factor $S$.

$$
p_{\text {coll }, S}=1-e^{-2 G_{S}} \text {. }
$$

The transmission time of a packet $T_{S}$ in LoRa is given by Eq. 4 with $L$ the length of the packet in bits and $R_{b}$ the bit rate from Eq. 1.

$$
T_{S}=\frac{L}{R_{b}} .
$$

And the amount of traffic generated per unit of time $\lambda$ is given by Eq. 5 with $N$ the number of nodes and $T_{i}$ the average packet interarrival time per node:

$$
\lambda=\frac{N}{T_{i}}[\text { packets } / \text { second }] .
$$

The amount of traffic $G_{S}$ generated during the transmission of one packet using spreading factor $S$ is:

$$
G_{S}=\lambda p_{S} T_{S}[\text { packets / transmission time using } S],
$$

where $p_{S}$ is the fraction of nodes using spreading factor $S$, which is the variable used to optimize the packet error rate. Notice that the unit of $G_{S}$ is different from $\lambda$ as we are not interested how many packets are generated per unit of time but rather during the transmission of a packet. Combining Equations 3, 4 and 6 gives $p_{\text {coll }, S}$ :

$$
p_{\text {coll }, S}=1-e^{-\left[\frac{2^{S+1}}{S} \frac{L}{B} p_{S} \lambda\right]} .
$$

b) Collisions with different spreading factors: The second loss of packets is due to a low SINR. One important cause of low SINR is collisions with different spreading factors. Packets suffering these collisions are only lost when the total interference is higher than the spreading gain. It is important to note that at least $-5 \mathrm{~dB}$ of SINR is needed for spreading factor 7 to receive packets, according to [13]. $-5 \mathrm{~dB}$ corresponds to more than 3 colliding packets with equal received power. Even more colliding packets (i.e. a lower SINR) are needed for higher spreading factors because they have a higher co-channel interference rejection (CIR). The authors in [14] presented a $16 \mathrm{~dB}$ CIR for spreading factor 7 from simulations allowing a lot more colliding packets before losing a packet. Therefore, we assume that this type of interference is not important when considering unconstrained power control.

Fairness Optimization: To maximize the fairness, the maximal collision rate with the same spreading factor, shown in Eq. 7, should be minimized:

$$
\min \max _{S} p_{c o l l, S} \text {. }
$$

This optimization is constraint as that the sum of all probabilities should be unity (Eq. 9), because each node has exactly one spreading factor.

$$
\sum_{S=7}^{12} p_{S}=1
$$

If we solve Eq. 8 to have a minimal overall collision probability, the result gives Eq. 10:

$$
p_{S}=\frac{S}{2^{S}} / \sum_{i=7}^{12} \frac{i}{2^{i}} \text {. }
$$

So, combining Eq. 10 and 7 results in Eq. 11:

$$
p_{\text {coll }, S}=1-e^{\frac{-2 l C_{\text {total }}}{B \sum_{i=7}^{12} \frac{i}{2^{i}}}} .
$$

Notice that the optimal distribution $p_{S}$ results in a fair collision probability $p_{\text {coll }, S}$ as it is independent of the spreading factor and as a result, all nodes have the same collision probability.

\section{B. Distribution with discrete power control and limited range}

Perfect power control is infeasible due to the restricted and discrete power levels. The impact of this limitation is discussed in this subsection.

The solution to our problem with limited power options is challenging. With limited power options, the probability to use spreading factor $S, p_{S}$ not only depends on the spreading factors used by other nodes, but also on the distance to the base station, and other nodes transmit power. When $N$ nodes are considered, the search space for optimal spreading factor and power for each node is $N^{6 \times 5}$, to accommodate the 
6 different spreading factors and 5 different power settings available as described in Sec. II-B. This optimization problem can easily be solved by a genetic algorithm. This algorithm has been applied to different cell sizes each with 300 nodes. The genetic algorithm was configured with a population size of 200, a crossover factor of 0.8 , an elite count of 20 and 10000 generations.

The results of the genetic algorithm are presented in Fig. 1. This figure clearly shows the distribution of spreading factors over all nodes is identical to the distribution calculated in Sec. III-A, provided the maximal difference in path loss is below $30 \mathrm{~dB}$. Below $30 \mathrm{~dB}$, the spreading gain of CSS and the power control combined are able to provide the required SINR to all nodes. However, this is not the case when the difference in path loss is higher. A far node loses its packet if it collides with a packet from a node close to the base station, even if they have different spreading factors. As a result, close nodes minimize their impact on the overall network by using the lowest spreading factor. This spreading factor has the lowest impact as the time on air is shorter due to the higher bit rate. In this scenario, the effect of collisions with different spreading factors must not be ignored.

Eq. 7 clearly shows that higher usage of lower spreading factors also results in an overall higher collision probability as more packets use the same spreading factor. To reduce the maximal difference in path loss and subsequently, collision probability, we propose to split the traffic over different channels or frequencies. Each unique channel will be used by nodes that have similar path losses.

When looking at the spreading factor distribution and power control found by the genetic algorithm, the following four guidelines were extracted:

1) Nodes with the lowest path loss get the lowest spreading factor.

2) The distribution of spreading factors is identical to the distribution from Sec. III.

3) The sum of received power and CIR should be higher than the highest received power of a node with a different spreading factor.

4) If the previous rule is impossible, the node with the lowest path loss will get the lowest spreading factor and the lowest power. The other nodes form a new group. This new group should again follow these guidelines.

We also noticed that the node with the highest packet error rate was the node with the highest path loss and spreading factor 8 . The reason for this higher error rate is the slower increase in path loss far from the base station. So, nodes far from the base station can more easily cope with this increase in path loss with their CIR than the nodes with spreading factor 8 closer to the base station. The optimal distribution of spreading factors in Fig. 1 can be found if we give all nodes that can corrupt this nodes' packets spreading factor 7 and power $2 \mathrm{dBm}$ (i.e. the weakest settings as the shortest packets and the lowest power) and scale the distribution derived in Sec. III for all the other nodes.

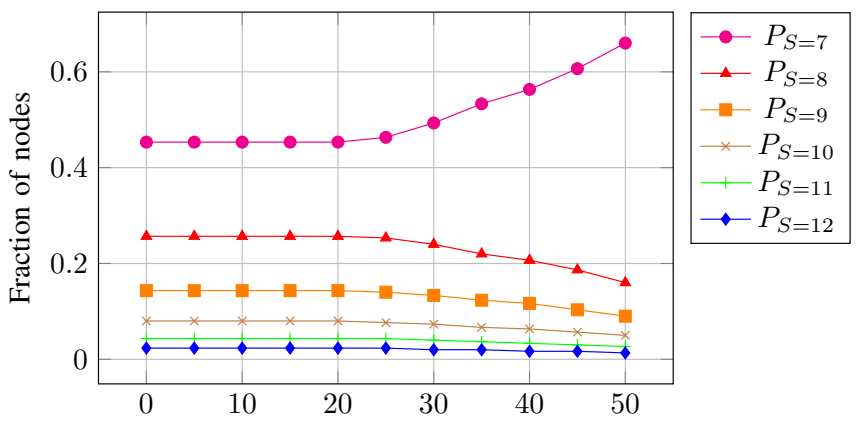

Maximal difference between maximal and minimal path loss

Fig. 1. Fraction of nodes to use a defined spreading factor versus the difference between the minimal and maximal path loss in $\mathrm{dB}$. From $25 \mathrm{~dB}$, the distribution changes as far nodes will collide with the closest nodes even with different spreading factors.

\section{THE PROPOSED SCHEME}

Given the insights from Sec. III, we present a scheme to determine the optimal configuration of the network, specified in Algorithm 1. This algorithm should be executed at regular intervals or at certain events, e.g. a new sensor is connected.

The algorithm starts by sorting the nodes by the distance to the base station or equivalently, by their estimated path loss (line 4). The estimated path loss is their received power minus the transmit power assigned by the base station. Next, the list is split in $K$ groups and a different channel is assigned to each group (line 5-9). This limits the difference in path loss between the node closest to the base station and the node furthest from the base station in that group, as proposed in Sec. III-B. The number of channels $K$ is limited by the network.

When all nodes are part of a group, nodes get a spreading factor from the distribution derived in Sec. III (line 10-13) (guideline 2) where nodes closest to the base station get the lowest spreading factor (guideline 1). To verify whether this is a good distribution, the link budget (i.e., received power and CIR) of the last node with spreading factor 8 (node with the highest path loss and spreading factor 8) is calculated (line 15-18). Here, as a feasibility check, the most extreme values will be applied to verify if the proposed distribution is possible (guideline 3). If the resulting link budget for the considered node is better than the received power of the fist node in the group, then it is possible to obtain a balanced power control and the power can be allocated (line 23-29).

If there is no solution (guideline 4), the lowest values are assigned to the first node and this node is removed from the list (line 19). The algorithm assigns new spreading factors and checks the feasibility of the new distribution, as described above (line 10-20). The algorithm repeats these steps until a valid power configuration has been found. Then, the algorithm determines the power of all remaining nodes in the group. If a node has spreading factor 7 , the algorithm makes sure that the sum of received power and CIR is higher than the received power of the first node with spreading factor 8 (i.e. the transmit power $P_{j=J, k}$ and the path loss $P_{L O S S, j=J, k}$ ) (line 21-22). Else, with a different spreading factor, the algorithm makes 


\begin{tabular}{|l|l|l|l|}
\hline Parameter & Value & Unit & Comments \\
\hline \hline$R$ & 1000 & $\mathrm{~m}$ & Radius of the cell \\
\hline$N$ & 1000 & & Number of nodes \\
\hline$K$ & 3 & & Number of channels \\
\hline$T_{i}$ & 600 & $\mathrm{~s}$ & $\begin{array}{l}\text { Average time between } 2 \\
\text { packet arrivals }\end{array}$ \\
\hline$L$ & 85 & byte & Total length of a packet \\
\hline$P_{0}$ & 40 & $\mathrm{~dB}$ & Initial power loss \\
\hline$n_{P L}$ & 4 & & Path loss exponent \\
\hline$E(X)$ & 0 & $\mathrm{~dB}$ & Mean value of shadowing \\
\hline $\operatorname{var}(X)$ & 2 & $\mathrm{~dB}$ & Variance of shadowing \\
\hline
\end{tabular}

TABLE III

PARAMETERS OF THE SIMULATION.

sure that the sum of received power and CIR is higher than the received power of the first node still in the list (i.e. the transmit power $P_{j=1, k}$ and the path loss $P_{L O S S, j=1, k}$ ) (line 23-29) (guideline 2). By iterating over the list backwards, we can update the power of the nodes with spreading factor 8 first and then update the nodes with spreading factor 7 .

One important aspect to take into account is the complexity of the algorithm. LPWANs can connect lots of nodes, so the algorithm should be computationally cheap. The complexity of the scheme proposed in Alg. 1 is $O\left(N^{2} / K\right)$ where $K$ is the number of different channels that can be used and $N$ the number of nodes. Although this looks high, it should be noted that the double loop is not executed every time. If the difference between the highest path loss and lowest path loss is limited to $30 \mathrm{~dB}$, there is no inner loop in this algorithm, leading to a complexity of only $O(N / K)$ for each group. The latter is generally the case for the group far from the base station.

\section{EVALUATION AND RESUlts}

To validate the proposed algorithm, it is implemented in ns-3 [15]. As a reference for comparison, a different scheme based on the link budget of each node is also applied as has been used in [4]. This second scheme allocates the lowest spreading factor and lowest power to each node, as all nodes are able to reach the base station as stated in Sec. II. The nodes can however randomly select a channel, which mixes far and close nodes. This mixure results in unbalanced power and link budgets. Using 3 channels randomly also means that the load on each channel will be comparable in both the proposed scheme and the distance based scheme. The parameters of the simulation are shown in table III.

The results of the simulation are summarized in Fig. 2. This figure shows the average packet error ratio (PER) for nodes at a given distance from the base station. The figure shows that close to the base station, there is no problem for either scheme. But, going further away from the base station, the performance drops until the random access limit is reached. The PER for this limit in the distance based scheme is $12 \%$. For our proposed scheme, Fig. 2 shows clearly an

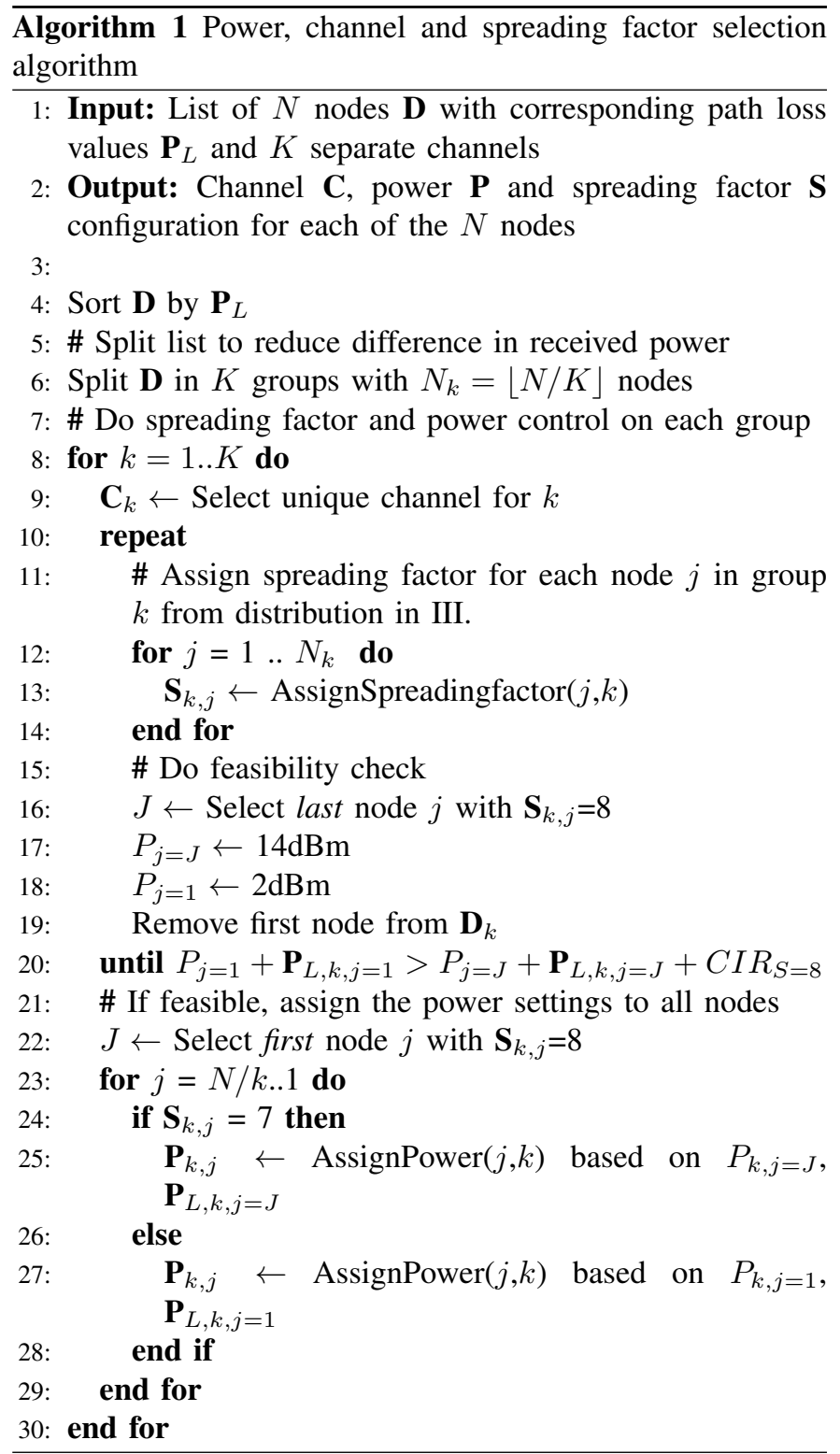

improvement for nodes far from the base station, the primary goal of this work. The packet error ratio in this region goes down to $6 \%$. This result is the random access limit of the network due to the limited amount of spreading factors. When looking at the overall network PER, we notice that also this value has decreased with almost $42 \%$.

The figure also shows that there is an increase of packet errors closer to the base station. While before, the probability of colliding with a closer node was small, now, it has increased because all this traffic is grouped on the same channel. The figure shows a higher PER around 350 meters from the base station. All node at this distance are part of the first group. The higher PER is a result of the larger path loss difference in the group closest to the base station compared to the groups further from the base station. Sec. III-B showed that it was impossible to keep the same spreading factor distribution with larger differences in path loss. The maximum in the figure 


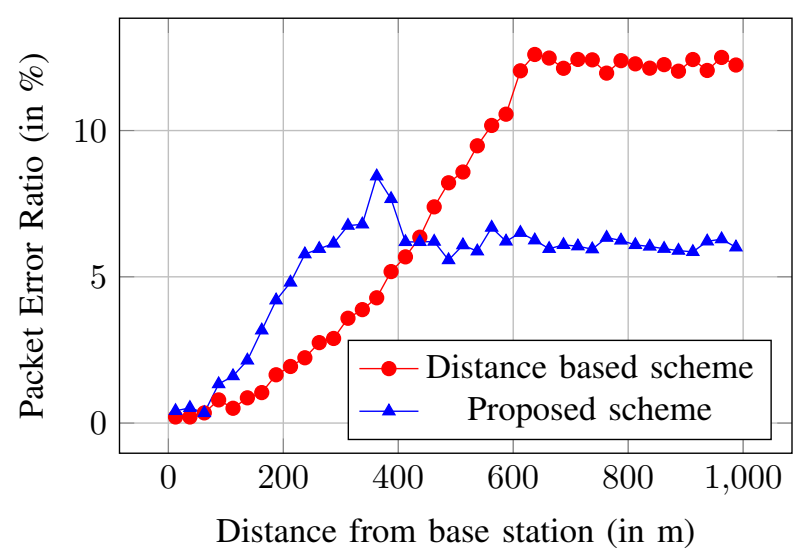

Fig. 2. The proposed scheme gives a significant improvement for the packet error ratio when nodes are far from the base station. The fairness in this network is higher than the SoA scheme.

is the result of the large difference in path loss between the nodes in the first group. The nodes in this group experience more collisions, because of nodes close to the base station. This problem could be solved by moving some of the nodes to other channels to balance the collision probability over the other groups

The above experiment is repeated for 100 to 900 nodes in a cell, with smaller packets than above. In Fig. 3, the PER for the edge users is shown under these circumstances. The edge users are defined as the $10 \%$ furthest nodes. The figure shows that the previous simulation results can be generalized to different loads. For all loads, the PER is decreased up to $50 \%$ and this result remains constant. The algorithm comes with a cost though: the longer transmission times consume more energy and also the higher transmit power draws more power than the distance based scheme.

For the results, it should be noted that due to restriction in memory of the pc, the maximum amount of nodes was 1000 . All the parameters for transmission need to be broadcasted to all the nodes, leading to a $O\left(N^{2}\right)$ memory consumption. But, it is assumed that the trend of reducing the PER can also be achieved for higher loads of the network.

\section{CONCLUSION}

This paper proposed a scheme for power and spreading factor allocation in long range networks. The goal was to optimize the packet error rate for the users far from the base station and, as a result, to make these networks more fair. To get to this result, first, the optimal distribution of spreading factors was taken into consideration. We have shown that there exists an optimal solution when there is perfect power control and an analysis has been given what happens if this control is discrete and limited. Based on these results, our scheme for spreading factor distribution was proposed. The desire was to develop an efficient scheme to control possibly many nodes in the network. Finally, simulation results showed that an improvement of almost $50 \%$ in packet error rate for edge users could be achieved with good power and spreading factor

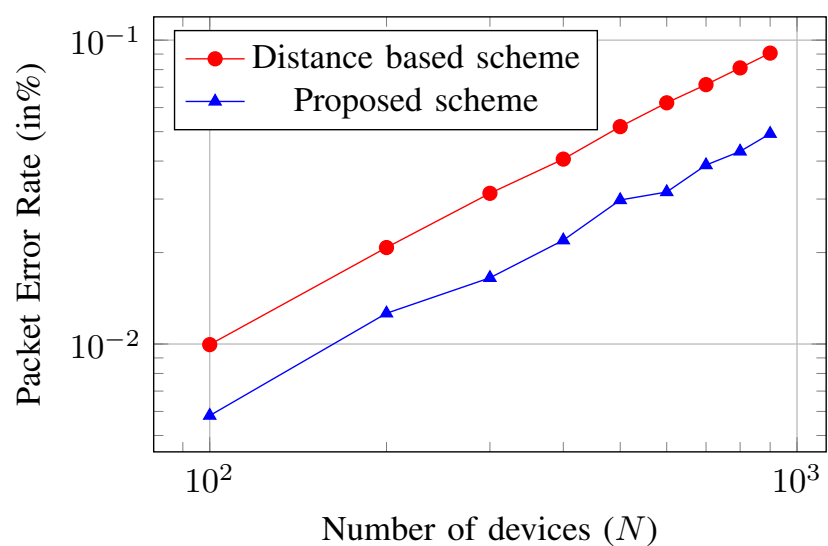

Fig. 3. The packet error rate for the last $10 \%$ of devices in the network for different loads of the network. The proposed scheme gives in all situations an improvement up to $50 \%$.

control. The overall network packet error rate was decreased by $42 \%$ with the proposed algorithm.

\section{ACKNOWLEDGMENT}

This work is supported by IWT CELTIC O\&O project ASUA/RoCCS.

\section{REFERENCES}

[1] T. T. Network, "The things network," http://ttn.org, 2016, accessed: 2016-03.

[2] N.Sornin, M. Luis, T. Eirich, T. Kramp, and O. Hersent, LoRaWAN Specification, January 2015. [Online]. Available: http://www.lora-alliance.org

[3] B. Reynders, W. Meert, and S. Pollin, "Range and coexistence analysis of long range unlicensed communication," in $201623 \mathrm{rd}$ International Conference on Telecommunications (ICT) (ICT 2016), Thessaloniki, Greece, May 2016.

[4] O. Georgiou and U. Raza, "Low power wide area network analysis: Can lora scale?" arXiv preprint arXiv:1610.04793, 2016.

[5] A. Augustin, J. Yi, T. Clausen, and W. M. Townsley, "A study of lora: Long range \& low power networks for the internet of things," Sensors, vol. 16, no. 9, p. 1466, 2016.

[6] F. Adelantado, X. Vilajosana, P. Tuset-Peiró, B. Martínez, and J. Melià, "Understanding the limits of lorawan," CoRR, vol. abs/1607.08011, 2016. [Online]. Available: http://arxiv.org/abs/1607.08011

[7] Z. Rosberg, "Transmitter power control with adaptive safety margins based on duration outage," Wireless Personal Communications, vol. 19, no. 1, pp. 81-90, Oct 2001. [Online]. Available: http: //dx.doi.org/10.1023/A:1011953803199

[8] D. Sylvia, B. Jothi Mohan, and D. Srinivasa Rao, "Power control in wireless networks: A survey," constraints, vol. 56, p. 57.

[9] P. N. Pathirana, "Location based power control for mobile devices in a cellular network," in TENCON 20052005 IEEE Region 10, Nov 2005, pp. 1-6.

[10] H. S. Dhillon, H. Huang, H. Viswanathan, and R. A. Valenzuela, "Fundamentals of throughput maximization with random arrivals for $\mathrm{m} 2 \mathrm{~m}$ communications," IEEE Transactions on Communications, vol. 62, no. 11, pp. 4094-4109, Nov 2014.

[11] Semtech, "Lora - semtech," http://iot.semtech.com, 2016, accessed: 2016-03.

[12] A. S. Tanenbaum, "Computer networks. 5th," 2011.

[13] $860 \mathrm{MHz}$ to $1020 \mathrm{MHz}$ Low Power Long Range Transceiver, Semtech Corporation, Jul. 2014, rev. 2.

[14] C. Goursaud and J.-M. Gorce, "Dedicated networks for IoT PHY / MAC state of the art and challenges," EAI endorsed transactions on Internet of Things, Oct. 2015. [Online]. Available: https://hal.archives-ouvertes.fr/hal-01231221

[15] Ns-3, "ns-3," http://nsnam.org, 2015, accessed: 2016-03. 